\title{
NEURONAVIGATION-GUIDED VERSUS FLUOROSCOPIC-GUIDED MICRONEUROSURGICAL TRANSSPHENOIDAL PITUITARY SURGERY
}

\author{
Sharad Pandey1, Anurag Sahu², Himanshu Jain ${ }^{3}$, Kulwant Singh ${ }^{4}$, Vivek Sharma ${ }^{5}$
}

${ }_{1}^{1}$ Assistant Professor, Department of Neurosurgery, Dr. RML PGIMER, New Delhi, India.

${ }^{2}$ Assistant Professor, Department of Neurosurgery, IMS, BHU, Varanasi, Uttar Pradesh, India.

${ }^{3}$ Consultant Neurosurgeon, Anand Hospital, Kurkshetra, India.

${ }_{4}^{4}$ Professor and HOD, Department of Neurosurgery, IMS, BHU, Varanasi, Uttar Pradesh, India.

${ }^{5}$ Ex-Professor, Department of Neurosurgery, IMS, BHU, Varanasi, Uttar Pradesh, India.

\section{BACKGROUND}

The transsphenoidal route for resection of pituitary adenomas eliminates the need for craniotomy to expose the pituitary gland. It creates narrow access to a deep region of the skull rich with critical neurovascular structures. To ensure an appropriate trajectory, intraoperative image-guidance has been used in transsphenoidal surgeries for decades. The evolution of image-guidance in pituitary surgery has included the use of intraoperative fluoroscopy, CT, ultrasound and MRI. The neuronavigational systems have been used successfully in the setting of pituitary adenoma resection, but comparative analysis with commonly available fluoroscopic-guided surgery is lacking.

The objective of this study is to compare the effectiveness and significance of neuronavigation vs fluoroscopy in transsphenoidal microneurosurgical removal of pituitary adenoma.

\section{MATERIALS AND METHODS}

This is a non-randomised controlled trial. Data of patients who were surgically treated for a pituitary lesion at the Department of Neurosurgery between May 2012 and May 2017 was collected. Age, sex, race, presenting symptoms, length of hospital stay, surgical approach, duration of surgery, tumour pathological features, Gross Total Resection (GTR) of tumour, recurrence of the lesion and intraoperative and postoperative complications were noted. All procedures were performed by the neurosurgical team, who were initially unfamiliar with the neuronavigation approach and therefore used traditional fluoroscopy.

\section{RESULTS}

30 patients who underwent fluoroscopic-guided and 30 patients who underwent neuronavigation-guided microsurgical removal of pituitary were included in the study. The mean time taken during surgery was significantly less $(\mathrm{p}<0.001)$ in neuronavigation group as compared to the fluoroscopy group. Also, there was significantly less amount of intraoperative blood loss in neuronavigation group. Postoperative residual tumour volume as assessed by a postoperative CT scan was less than $0.5 \mathrm{~mL}$ in $67 \%$ patients in neuronavigation group, while it was between $1-1.5 \mathrm{~mL}$ in $60 \%$ patients with fluoroscopy. Other postoperative complications like diabetes insipidus, CSF rhinorrhoea, meningitis, vision loss and sinusitis were comparable between the two groups.

\section{CONCLUSION}

The application of neuronavigation in the transsphenoidal surgery for resection of pituitary adenoma can improve the safety of transsphenoidal approach, decreases surgical complications and duration of surgery.

\section{KEY WORDS}

Neuronavigation; Fluoroscopy; Pituitary Adenoma; Transsphenoidal Approach.

HOW TO CITE THIS ARTICLE: Pandey S, Sahu A, Jain H, et al. Neuronavigation-guided versus fluoroscopic-guided microneurosurgical transsphenoidal pituitary surgery. J. Evolution Med. Dent. Sci. 2018;7(28):3238-3242, DOI: $10.14260 /$ jemds/2018/728

\section{BACKGROUND}

The transsphenoidal approach is accepted as the procedure of choice for the surgical management of pituitary adenomas. Although, it is possible to rely solely on anatomic landmarks to reach the sphenoid sinus and sella, still intraoperative imaging is used by most surgeons as an integral part of the transsphenoidal approach.

'Financial or Other Competing Interest': None.

Submission 09-06-2018, Peer Review 27-06-2018,

Acceptance 29-06-2018, Published 09-07-2018.

Corresponding Author:

Dr. Anurag Sahu,

Assistant Professor

Department of Neurosurgery,

IMS, BHU, Varanasi, Uttar Pradesh, India.

E-mail: anuragsahu25@rediffmail.com

DOI: $10.14260 /$ jemds/2018/728
Image guidance in pituitary surgery began with the use of intraoperative air encephalography and C-arm videofluoroscopy and continues to expand with the addition of newer techniques such as intraoperative ultrasound, computer-based neuronavigation, intraoperative MRI and endoscopic-assisted surgery (Chung1, De Lara²).

As these techniques to reduce surgical morbidity enhances operative success and improved post-operative recovery continue to evolve, surgeons need to recognise the benefits and limitations of the various technologies and select the appropriate neuronavigation tool for individual cases (Samip ${ }^{3}$.

This study has been conducted to compare the efficacy of neuronavigation over videofluoroscopy in transsphenoidal pituitary surgery. 


\section{MATERIALS AND METHODS}

In this study we compared the intraoperative and immediate postoperative complications among the two most widely used methods of intraoperative imaging, namely fluoroscopy and neuronavigation. We did non-randomised controlled trial in the Department of Neurosurgery, SSL Hospital, Institute of Medical Sciences, Banaras Hindu University over a period of five years (2012 - 17). A total of 60 patients of MRI/ CT proven pituitary macroadenoma and pituitary microadenoma cases, in which medical treatment had failed and surgery was done was taken for convenience. Age, sex, race, presenting symptoms, length of hospital stay, surgical approach, duration of surgery, tumour pathological features, Gross Total Resection (GTR) of tumour, recurrence of the lesion and intraoperative and postoperative complications were noted. All procedures were performed by the neurosurgical team, who was initially unfamiliar with the neuronavigation approach and therefore used traditional fluoroscopy. Such selected patients were divided non-randomly into two groups.

\section{Group A}

30 patients in whom intraoperative neuronavigation was used for localisation of sphenoid sinus and sella turcica during transsphenoidal surgery for removal of pituitary tumour.

\section{Group B}

In 30 patients, conventional fluoroscopy was used intraoperatively for localisation of sphenoid sinus and sella turcica.

In Group A, contrast enhanced CT scan of the patients was taken in neuronavigation protocol one day prior to surgery. The data was stored in NR CD/ DVD in DICOM format and was loaded on Treon plus Stealth Station Medtronic.

In Group A patients, tracer registration was done prior to giving the surgical incision. All patients in both groups underwent transsphenoidal removal of pituitary adenomas using identical surgical techniques with an operating microscope.

In Group A patient, all the internal bony landmarks were identified in respect to position of carotid arteries, sellar floor, anterior fossa floor and clivus correlating the operative anatomy with imaging studies and navigational adjuncts to ensure that the appropriate midline trajectory was maintained.

In Group B patients, bony landmarks are identified visually using a radio-opaque marker using fluoroscopy.

Duration of surgery was noted for each case from the time of gingival incision to closure. Intraoperative blood loss was estimated by measuring the exact amount of fluid in the suction bottle and by deducting from it the known amount of saline used for irrigation purpose.

Postoperatively, all the patients were shifted to neurosurgical ICU for elective ventilation. They were extubated the next morning. A postoperative CT scan (plain and contrast enhanced) of head was done for assessment of residual tumour volume/ haematoma in all patients.

Endonasal packing was removed after 48 hours and patients were allowed orally. Patients were followed up in the postoperative period for any complications. Patients were discharged when stable and again followed-up after 2 weeks.

\section{Statistical Methods}

Using IBM Statistical Package for Social Sciences (SPSS) 16 software, the Chi-square and Student's t-tests were used to measure the association of categorical data and means between the two groups. Relative risk reduction was used to measure the efficiency of implantation, blood loss and revision surgery when the neuronavigation system was used. A 'p' value $<0.05$ was considered significant. Mean scores obtained by each group were used for statistical analysis

\section{RESULTS}

Both the groups were similar in demographic variables. The mean age of patients in Group A was 33.67 years and in Group B was 33.34 years. There was no significant sex predilection in patients of pituitary tumours. Mean preoperative tumour volume was $6.167 \mathrm{~mL}$ in Group A and $6.812 \mathrm{~mL}$ in Group B. Both the groups were similar in tumour extension and invasion. The total operating room time includes the time required for the initial setup of the machine and tracer registration along with the actual surgical time (incision to closure). The mean operating time in Group A was 157 mins and in Group B was 282 mins, which shows a significant difference $(\mathrm{p}<0.001)$ (Table 1$)$.

System related errors like registration error happened only in Group A (10\%), as they are related to the neuronavigation machine. There was a significant reduction in intraoperative blood loss in Group A. The major reason behind this difference could be attributed to the prolonged surgery in Group B patients. Intraoperative CSF leak was seen in six $(20 \%)$ cases in Group A, while in four $(13.3 \%)$ cases in Group B (Table 2). The mean postoperative residual tumour volume in Group A was $0.45 \mathrm{~mL}$ and in Group B was $1.2 \mathrm{~mL}$ $(\mathrm{p}<0.001)$ (Table 3). The postoperative complications found in some of the patients were diabetes insipidus (transient), CSF rhinorrhoea, meningitis, vision loss and sinusitis (Table 4). However, the difference in postoperative complication rate was not statistically significant between two groups.

\begin{tabular}{|c|c|c|}
\hline & Group A & Group B \\
\hline Upto 2 hours & 1 & 0 \\
\hline 2-3 hours & 25 & 0 \\
\hline 3-4 hours & 4 & 2 \\
\hline 4-5 hours & 0 & 20 \\
\hline$>5$ hours & 0 & 8 \\
\hline
\end{tabular}

Fisher's Exact Test: $\mathrm{p}<0.001$

\begin{tabular}{|c|c|c|c|}
\hline & Group A & Group B & P-value \\
\hline System related errors & 3 & 0 & 0.237 \\
\hline Haemorrhage & & & \\
$<100 \mathrm{~mL}$ & 25 & 5 & $<0.001$ \\
$>100 \mathrm{~mL}$ & 5 & 25 & \\
\hline CSF leak & 6 & 4 & 0.217 \\
\hline \multicolumn{3}{|c|}{ Table 2. Intraoperative Complication } \\
\hline
\end{tabular}

\begin{tabular}{|c|c|c|}
\hline $\begin{array}{c}\text { Tumour Volume } \\
\text { (mL) }\end{array}$ & Group A & Group B \\
\hline $0-0.5$ & 20 & 3 \\
\hline $0.5-1.0$ & 8 & 4 \\
\hline $1.0-1.5$ & 2 & 18 \\
\hline $1.5-2.0$ & 0 & 3 \\
\hline$>2.0$ & 0 & 2 \\
\hline Table 3. Postoperative residual Tumour Volume \\
\hline
\end{tabular}

Fisher's Exact Test: $\mathrm{p}<0.001$ 


\begin{tabular}{|c|c|c|}
\hline & Group A & Group B \\
\hline Diabetes insipidus (transient) & 4 & 2 \\
\hline CSF rhinorrhoea & 3 & 3 \\
\hline Meningitis & 0 & 3 \\
\hline Vision loss & 0 & 2 \\
\hline Sinusitis & 1 & 3 \\
\hline \multicolumn{2}{|c|}{ Table 4. Postoperative Complications } \\
\hline
\end{tabular}

$\mathrm{p}=0.176$

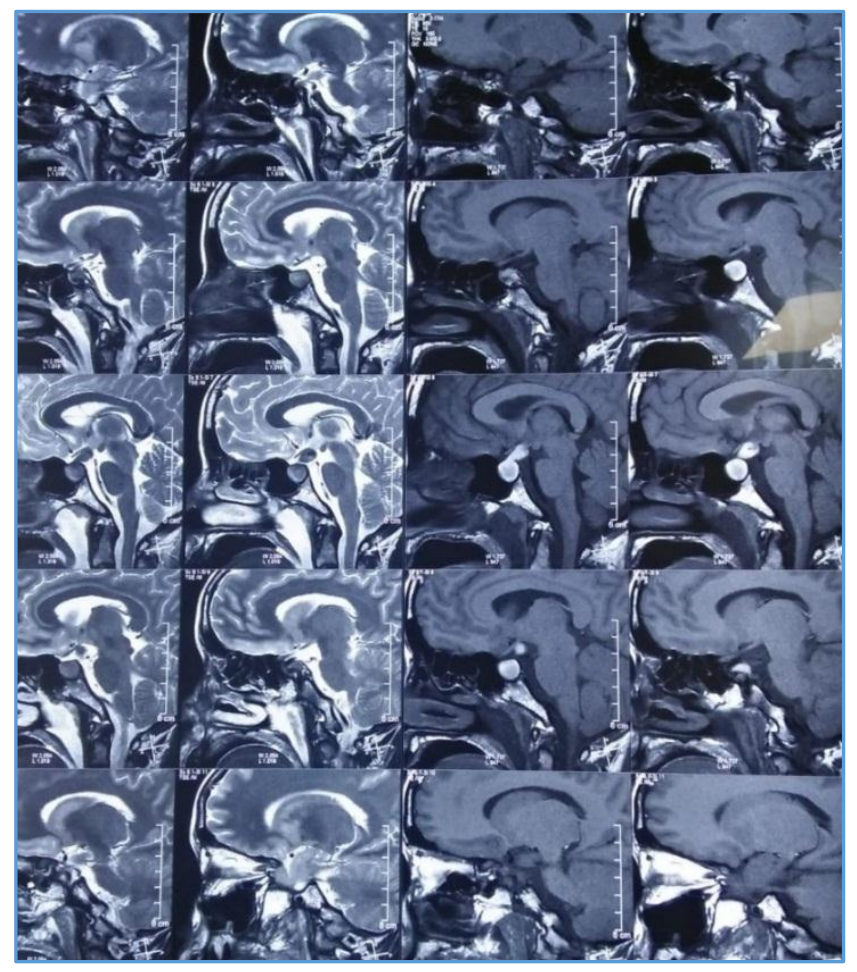

Figure 1. T1 and T2 Weighted Sagittal MRI Brain images showing Pituitary Adenoma

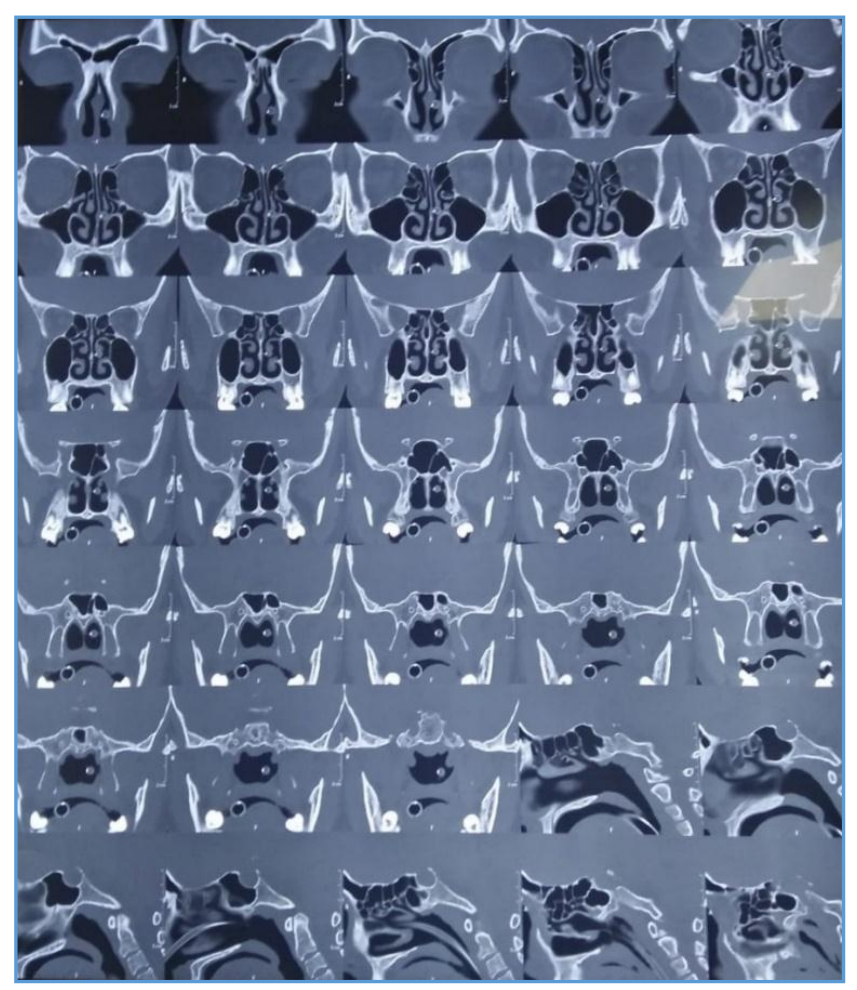

Figure 2. CT PNS Coronal and Sagittal images showing Location of Sella

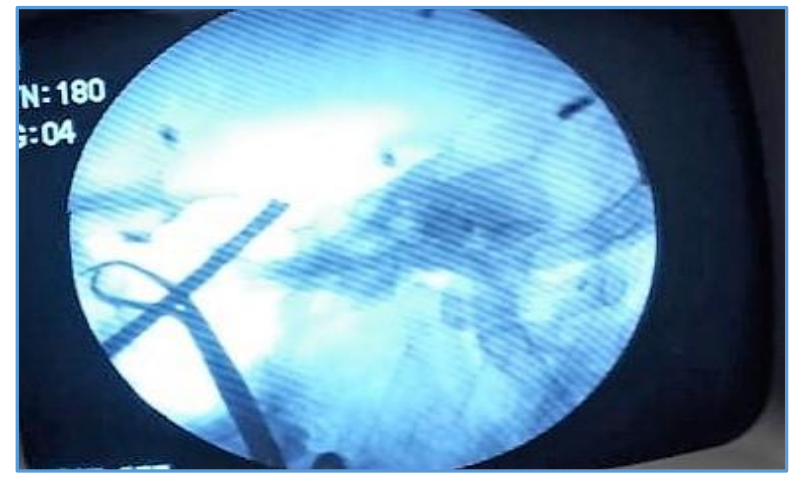

Figure 3. Intraoperative FluoroscopicGuided Localisation of Pituitary Sella

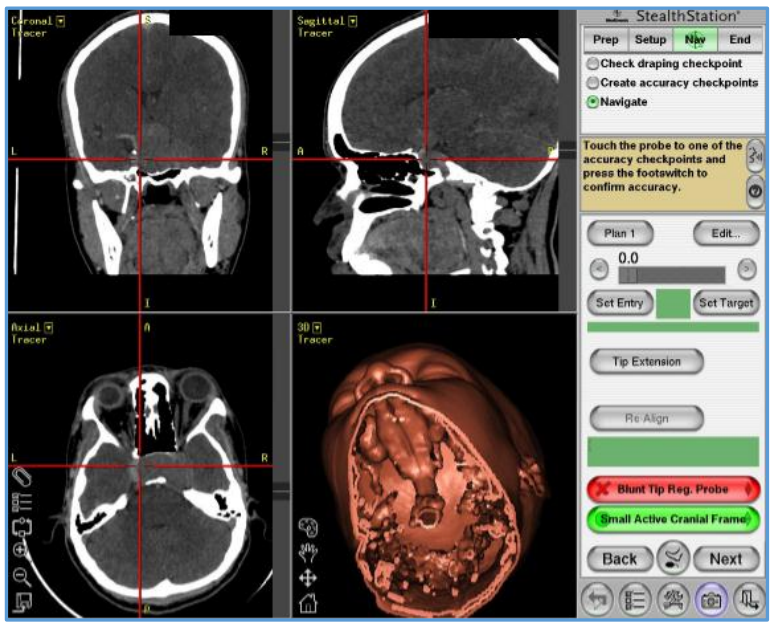

Figure 4. Intraoperative Neuronavigation-Guided Localisation of Pituitary Adenoma and Sella

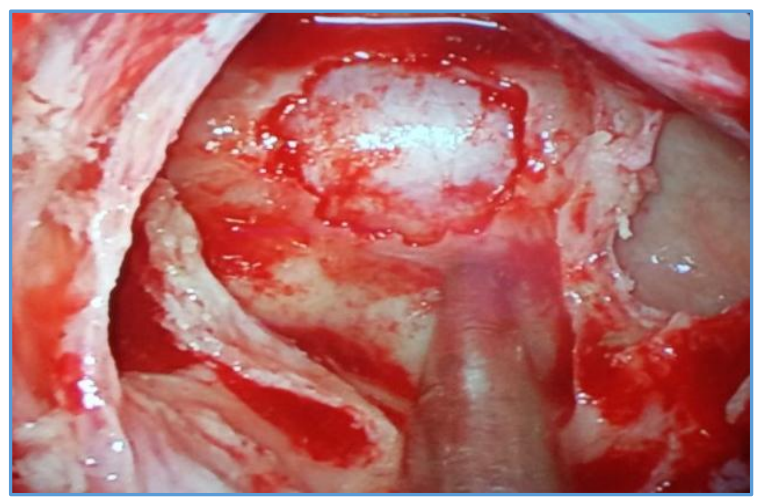

Figure 5. Intraoperative image showing Sella Floor with Intersinus Septum and Dura

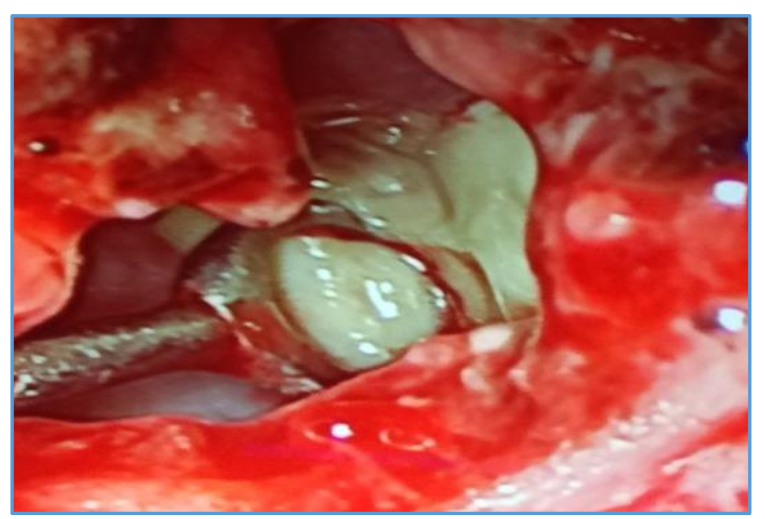

Figure 6. Intraoperative image showing Removal of Pituitary Adenoma using Ring Curette 


\section{DISCUSSION}

The most widely used intraoperative imaging device is the $\mathrm{C}$-arm video fluoroscope. The $\mathrm{C}$-arm video fluoroscope is sufficient for most routine first time transsphenoidal operations, where tumour is confined to the sella. Nevertheless, there are limitations. Fluoroscopy does not provide direct view of the pituitary gland nor of the adjacent brain parenchyma and cranial nerves. Also, it cannot direct the surgeon regarding the laterality of these tumours. Another of its disadvantage is the radiation exposure (Walker ${ }^{4}$ ). Any intraoperative rotational adjustment of the head for an improved surgical view point requires a concurrent adjustment in the C-arm angle to maintain a true lateral image. It is ineffective in demonstrating the midline in the antero-posterior view and its use for this intraoperatively is limited due to microscope positioning conflicts and disruption of surgical access to the operative corridor (Wong5, De Lara²).

The current study focuses on the more recent and advanced method of intraoperative imaging that is neuronavigation and describes its advantageous role in transsphenoidal pituitary surgery for pituitary tumours. It provides a patient specific, three-dimensional (3-D) anatomy for intraoperative navigation, thus helping the surgeon to perform complicated procedures with improved accuracy and safety. Thus, neuronavigation adds an entirely new dimension to neurosurgery (Orringer6).

In our study we measured the outcome of microscopic transsphenoidal pituitary surgery along several parameters, first and foremost of which were complications related to surgery.

Most of the morbidity arises from damage to neurovascular structures in or proximal to the surgical field. These structures include both the anterior and posterior lobes of the normal pituitary gland, optic chiasma, cavernous sinuses and carotid arteries. Damage to these structures can in general be entirely avoided with scrupulous and meticulous surgical technique and an accurate degree of localisation of tumour.

The duration of surgery is also of utmost importance. The complications heralded by the prolonged surgery are obvious. It leads to increased morbidity due to prolonged exposure to anaesthesia and more blood loss. The shortened duration of procedure in Group A is due to the easy and accurate localisation of the sella turcica and sphenoid sinus, which facilitates tumour removal. The initial registration step in Group A takes only 5 - 8 minutes and thus does not prolong the total time duration of the surgery.

Another disadvantage of intraoperative fluoroscopy is that the patient, surgeon and operating room personnel are exposed to ionising radiation, often necessitating the use of cumbersome lead aprons throughout the case.

We followed the patients in our study during their postoperative hospital stay and further when they came for follow-up after two weeks. In Group A, four patients had transient diabetes insipidus and three patients had CSF rhinorrhoea. Sinusitis was seen in only one patient on follow-up, whereas in Group B two patients had transient diabetes insipidus, three patients had CSF rhinorrhoea, three had meningitis, two had vision loss and one had sinusitis. This significant difference in the postoperative complication rate further stresses upon the need of increased accuracy of tumour removal and minimal damage to the nearby structures during surgery.

We observed that CT-based neuronavigation definitely increases the direct cost for the patient, but the decreased surgical time and the markedly reduced postoperative complication rate more than compensates for it. The complications commonly seen in postoperative period in patients of Group B required further management in the form of advanced spectrum antibiotics, insertion of lumbar drain, increased hospital stay etc. which indirectly adds to the total cost of the procedure.

\section{CONCLUSION}

Our study has stressed upon the importance of accuracy of localisation of tumour margins during pituitary surgery. The ease and quickness with which sella is precisely located using neuronavigation proved to be a boon in transsphenoidal surgery. It definitely reduced the operating time, but not at the cost of increased complications. The advantages conferred like accuracy and completeness of excision and decreased postoperative morbidity far outweighed the disadvantages like increased cost and training (Carvi Nievas ${ }^{7}$ ). Neuronavigation reduces surgical time, damage to surrounding structures thereby avoiding major intraoperative complications, decreases postoperative morbidity due to damage to nearby structures during surgery thus reducing the hospital stay (Slavin ${ }^{8}$ ). We also noticed a significant decrease in the residual tumour volume postoperatively.

The judicious use of appropriate resources based on the level of intraoperative guidance that will be required and an understanding of the relative utility and limitations of each modality will limit the superfluous use of advanced neuroimaging techniques. The use of intraoperative neuroimaging is not a replacement for surgical experience and a thorough knowledge of regional anatomy, but provides another tool by which the neurosurgeon can reduce the risk associated with surgical access and treatment of pituitary pathology.

\section{REFERENCES}

[1] Chung TK, Riley KO, Woodworth BA. The use of imageguidance during transsphenoidal pituitary surgery in the United States. American Journal of Rhinology \& Allergy 2015;29(3):215-20 doi:10.2500/ajra.2015.29.4166.

[2] De Lara D, Filho LFSD, Prevedello DM, et al. Application of image guidance in pituitary surgery. Surgical Neurology International 2012;3(Suppl 2):S73-S8. doi:10.4103/2152-7806.95418.

[3] Samip NP, Samy YA, Fernando LV, et al. Re-evaluation of the role of image guidance in minimally invasive pituitary surgery: benefits and outcomes. Computer Aided Surgery 2011;16(2):47-53. DOI: 10.3109/10929088.2011.552954

[4] Walker DG, Ohaegbulam C, Black PM. Frameless stereotaxy as an alternative to fluoroscopy for transsphenoidal surgery: use of the Insta Trak-3000 and a novel headset. Journal of Clinical Neuroscience 2002;9(3):294-7. 
[5] Wong GK, Poon WS, Ng SC, et al. Fluoroscopic frameless computer-assisted navigation for transsphenoidal surgery: a clinical assessment of accuracy in spatial position and trajectory. Minimally Invasive Neurosurgery 2004;47(1):29-31. DOI: 10.1055/s-2003-812535.

[6] Orringer DA, Golby A, Jolesz F. Neuronavigation in the surgical management of brain tumors: current and future trends. Expert Review of Medical Devices 2012;9(5):491-500. doi:10.1586/erd.12.42.
[7] Nievas CYMN, Höllerhage HG. Reliability of neuronavigation-assisted trans-sphenoidal tumor resections. Neurological Research 2007;29(6):557-62. 10.1179/016164107X164184.

[8] Slavin KV. Neuronavigation in neurosurgery: current state of affairs. Expert Review of Medical Devices 2008;5(1):1-3. 10.1586/17434440.5.1.1. 